94. Deutscher Röntgenkongress - 29. Mai bis 01. Juni 2013

\title{
Hellste Köpfe
}

Donnerstag, 30. Mai 2013, 15:30-17:30

$\nabla$

Hellste Köpfe I: Studieren \& Arbeiten im Ausland

Vorsitz: Dr. Diane Renz, Berlin, E-Mail: diane.renz@charite.de

15:30-15:45 Uhr Studieren im Ausland aus der Sicht eines weitgereisten Klinikchefs - Prof. Dr. med. Ulf Teichgräber E-Mail: ulf.teichgraeber@med.uni-jena.de

15:45-16:15 Uhr „Blick zurück nach vorn: Reminiszenzen an die Entwicklung der Neuroradiologie und Lehren aus einem unorthodoxen Werdegang“ Prof. Dr. Klaus Sartor

16:15-16:30 Uhr Expertenvortrag:

Famulaturbörse des BVMD - Christian Fischer Sill, Bundeskoordinator für den Famulantenaustausch E-Mailneo@bvmd.de

16:30-16:45 Uhr Erlebnisbericht I - PD Dr. Gerd Diederichs; Charité Universitätsmedizin Berlin, Institut für Radiologie, Charitéplatz 1, 10117 Berlin, E-Mail: gerd. diederichs@charite.de (Studienaufenthalt in San Francisco, Muskuloskelettalbildgebung)
16:45-17:00 Uhr Erlebnisbericht II - Dr Vera Fröling; Charité Universitätsmedizin Berlin, Institut für Radiologie, Charitéplatz 1, 10117 Berlin, E-Mail: vera.froeling@charite.de (PJ in China)

17:00-17:15 Uhr Erlebnisbericht III - Dr. Federico Collettini; Charité Universitätsmedizin Berlin, Institut für Radiologie, Charitéplatz 1, 10117 Berlin, E-Mail: federico.collettini@charite.de (gebürtiger Römer, seit 2010 Assistenzarzt an der Charité)

\section{7:15-17:30 Uhr Diskussion}

Samstag, 1. Juni 2013, 09:00-10:30 Uhr

Hellste Köpfe II: Röntgendiagnostik Live

Das Referenten-Team der Online-Kurse „Keine Angst vor Röntgenbildern im Hammerexamen " präsentiert und diskutiert interessante Fälle und ihre Bilder - ein Kurs zum Mitsehen, Mitreden und Mitlernen.

Referenten:

PD Dr. Rolf Janka, Erlangen

Dr. Florian Mayer, Münster

Prof. Dr. Michael Uder, Erlangen
Samstag, 01. Juni 2013, 14:15-15:30 Uhr $\nabla$

Hellste Köpfe IIII: Radiologie ist Zukunft

Vorsitz: Prof. Dr. Walter Gross-Fengels, EMail: gross-fengels@asklepios.com

14:15-14:30 Uhr Eingangsstatement: Radiologie ist Zukunft - Perspektiven des Fachs aus wissenschaftlicher und berufspolitischer Sicht - Prof. Dr. Walter Gross-Fengels

14:30-14:45 Uhr Herausforderung Kinderradiologie: Dr. Michael Buchholz (Lübeck),E-Mail: michael.buchholz@uk-sh.de

14:45-15:00 Uhr Schwerpunkt Neuroradiologie: Prof. Dr. Bernd Eckert (Hamburg), E-Mail: b.eckert@asklepios.com

15:00-15:15 Uhr Herzdiagnostik - ein Fall für die Radiologie - Prof. Dr. Matthias Gutberlet (Leipzig) E-Mail: matthias.gutberlet@herzzentrum-leipzig.de

15:15-15:30 Uhr Diskussion und Fragerunde 